\title{
PhiфBreast \& Theory of Spiral Cancer - New Diagnostic Techniques Breast Cancer Detection
}

\section{Ersilio Trapanese ( $\sim$ lio@fabtrapa.net)}

De Beaumont Bonelli Fondation https://orcid.org/0000-0003-1159-9322

\section{Giulio Tarro}

De Beaumont Bonelli Fondation

\section{Research Article}

Keywords: PhiథBreast, Theory of Spiral Cancer, Predictive Algorithm, Golden Ratio, Fibonacci Sequence, Gravity, Phyllotaxis, Breast Cancer

Posted Date: July 31st, 2021

DOl: https://doi.org/10.21203/rs.3.rs-714037/v1

License: (1) This work is licensed under a Creative Commons Attribution 4.0 International License. Read Full License 


\title{
PhiФBreast \& Theory of Spiral Cancer New Diagnostic Techniques Breast Cancer Detection
}

\author{
*Ersilio Trapanese Giulio Tarro
}

*Ersilio Trapanese

MD, Director from Division of Diagnostic Imaging \& Interventional Ultrasound - CMM Diagnostic Center - Cava de’ Tirreni, Italy

Member of the Board of Directors T. \& L. De Beaumont Bonelli Foundation for Cancer Research - Naples, Italy

Giulio Tarro

MD, PhD, President Foundation T. \& L. De Beaumont Bonelli for Cancer Research - Naples, Italy

*Corresponding author: Trapanese E, Member of the Board of Directors T. \& L. De Beaumont Bonelli Foundation for Cancer Research - Naples, Italy Tel: +390815463222; E-mail:lio@fabtrapa.net;

Email Id: lio@fabtrapa.net

Email Id: giuliotarro@gmail.com
ORCID Id (0000-0003-1159-9322)

ORCID Id (0000.0001-8183-4149) 


\begin{abstract}
Background: Breast cancer today is one of the most aggressive in women and new cases continue to increase worldwide. The incidence of this tumor is kept under control especially by surgery and to reduce mortality we need detect life threatening disease at an earlier stage.

We conducted two years a study for the identification and characterization of suspicious breast lesions using a new diagnostic technique applied to ultrasonography and mammography called "PhiథBreast."
\end{abstract}

Methods: Identification and characterization of category C4-C5 lesions of the breast with high Predictive Positive PPV value, by means of a new innovative method called "PhiФBreast" using the Golden Ratio (Phi, or $\Phi$ 1.618...) Fibonacci sequence and a Predictive Algorithm, applied to the ultrasonography and mammography with subsequent deepening with cytological examination using fine needle aspiration (FNAC), according to evaluation criteria of the Breast Imaging Report Data System (BI-RADS) and the American College of Radiology (ACR).

Usefulness of this research is to detect the breast cancer early on with this new diagnostic technique, in addition to develop a classification model of the histological type identified in the section areas and the percentage of probability in relation between the golden spiral and Fibonacci sequence.

This amazing intuition gave rise Theory of Spiral Cancer.

Results: Using Golden Ratio and Fibonacci sequence, applied to ultrasonography and mammography, we have experimented and developed a diagnostic map with characteristics of high probability of identifying suspicious lesions at an early stage.

We examined 987 women, 55 lesions detected with PhiФBreast pattern were classified according to BI-RADS descriptors for US-imaging, including morphologic features that had a high predictive value for the malignancy $(\mathrm{p}<0.001)$.

This innovative diagnostic tecnique has shown a sensitivity $95 \%$, a specificity $97 \%$, a positive predictive value $97 \%$, a negative predictive value $96 \%$.

The discriminating capacity of PhiФBreast was significantly better than normal ultrasonography $(\mathrm{P}<0,05)$.

Furthermore with a predictive algorithm associated with malignant cytology after FNAC, we have classified different types of cancer potentially life threatening for patients.

Conclusion: PhiФBreast could be an important new model diagnostic technique to be applied ultrasound and mammography for detection malignant lesions of category C4-C5.

In diagnostic imaging beyond to identification of a lesion and classification according to the BI-RADS category and the evaluation criteria of the ACR is fundamental recognize predictively the characteristics of an potentially aggressive tumor. Everything reinforces the concept that the early diagnosis is essential because it allows to remove small tumors and therefore capable of producing more limited metastases than the potential of the most voluminous neoplasms in order to plan an effective cure for the patient.

This new model (PhiФBreast) could represent the cornerstone for an important contribution of high science for the early diagnosis of Breast Cancer.

Keywords: PhiФBreast, Theory of Spiral Cancer, Predictive Algorithm, Golden Ratio, Fibonacci Sequence, Gravity; Phyllotaxis, Breast Cancer 


\section{Background}

Breast cancer represents the first cancer of frequency in women under 45 , with an even higher incidence in women up to 65 years. It affects 1 in 8 women over a lifetime and represents $29 \%$ of all female cancers and results in a mortality rate of $16 \%$.

The highest incidence is recorded in North America with 90 cases per 100,000 women [1,2].

In Europe, every year more than 200,000 women are affected by breast cancer, with an incidence ranging from 5 to $10 \%$ depending on the country. In Italy the incidence is mediumhigh. The cases of breast cancer diagnosed are around 31,000 a year, with an increasing incidence from the south to the north [3].

In the last 6 years the incidence of breast cancer has reached $13.8 \%$. There are several risk factors that contribute to the development of breast cancer. Most relevant non-changeable risk factors are: gender, age, genetic predisposition: mutations in the BRCA1, BRCA2 [4] genes (which carry a 60-90\% risk of developing a breast cancer over the course of life), $\mathrm{Li}$ Fraumeni [5] syndrome, mutation of the gene that codes for p53 [6], Lynch syndrome [7]. Ethnic group[8], atypical mammary hyperplasia, high breast density. Environmental factors: exposure to ionizing radiation in childhood and / or juvenile age (for example in women with previous Hodking lymphoma). Hormonal therapies: HRT [9] estroprogestinic, which involve a minimum increased risk. The purpose of this study is to identify a mammary neoplasm in early time using this new diagnostic technique, also to develop a classification model of the histological type identified in the various area of the Golden Ratio and the percentage of probability in relation between to the Fibonacci sequence.

Identification and characterization of category C4-C5 lesions of the breast with a high Positive Predictive PPV value, by means of a new revolutionary method called PhiФBreast using the Golden Ratio, Fibonacci sequence and a Predictive Algorithm, applied to ultrasonography and mammography with subsequent study with cytological examination using fine needle aspiration (FNAC) in respecting the criteria of the American College of Radiology (ACR) and Breast Imaging Report Data System (BI-RADS).

\section{Methods}

From January 2016 to february 2018, 987 consecutive patients, aged between 40 and 75 years, were enrolled for the ultrasound study of echostructure alterations in the breast.

All patients underwent a two-dimensional ultrasound examination (2 DUS) a Toshiba Aplio 500 ultrasound system, using high-frequency probes $13-15 \mathrm{MHz}$.

The carefully studied parameters of the lesions detected were the following: shape, echostructure, level of echogenicity, margins, size and topographical area.

On 55/987 patients were identified with PhiФBreast ultrasonographic study, solid lesions markedly hypoechoic echostructure, round shape with irregular and infiltrative margins and cuneiform shape with blurred margins (Fig. 1).

After obtaining informed consent, 55 selected patients underwent a mammography examination and subsequent diagnostic deepening by procedure Fine-Needle Aspiration Cytology (FNAC) under ultrasound guidance.

PhiФBreast imaging applied to the mammogram in the projections bilateral cranial-caudal (CC) and mediolateral oblique (MLO) has give rise a mapping of neoplastic nonpalpable breast lesions (Fig. 2 and Fig. 3).

PhiФBreast has produced data of great importance for the elaboration of a Predictive Algorithm on the probability of development of various histological types of tumors and percentage detectable in the areas of the Golden Ratio in relation to the Fibonacci sequence $(13,21,34,55,89,144)$ making use of a new nomenclature called “Trapanese's Section" (TS1,TS2,TS3,TS4,TS5,TS6) (Table 1).

The result of the cytology were identified 55 tumors: 13 ductal carcinomas in situ, 10 invasive ductal carcinomas, 6 invasive lobular tumors and 26 invasive carcinomas not otherwise specified.

Finally thanks to an intuition, the creativity and in depth studies, a theory was developed on the growth of spiral cancer cells called "Theory of Spiral Cancer".

Surgery was established based on the type of tumor identified in patients in the preoperative phase.

The histological diagnosis confirmed the tumor nature of the cells of the analyzed tissues, performed according to the criteria established by the American College of Radiology (ACR) and Reporting Breast Imaging and Data System (BI-RADS).

Patients underwent blood chemistry controls, tumor markers. The follow up period lasted about 1 year.

\section{Results}

\section{Theory of Spiral Cancer}

The Golden Ratio (Phi, or $\Phi$ 1.618...) is a potentially unifying quantity of structure and function in nature, as best observed in phyllotactic patterns in plants. For centuries, Phi $(\Phi)$ has been identified in human anatomy, and in recent decades, $\Phi$ has been prove in human physiology as well with scientific studies of some authors $[10,11]$.

It is possible, according to an intuition and in-depth studies on the Golden Ratio [12], Fibonacci Sequence [13] and Phyllotaxis [14], research from a discipline between botany and mathematics that breast cancer could have a model of growth and evolution that combines observation and the hypothesis according to in-depth scientific studies that we have carried out.

We believe that the breast cancer growth model could have a connection with the phyllotaxis and Fibonacci sequence, under influence of the gravity (g) [15].

The spirals are born from a growth property called selfsimilarity or scaling, that is the tendency to grow in size but preserving the same shape. However not all organisms grow in this similar way.

According to the theory of the spiral growth model of cancer, it is possible that the ab-initio lesion may mimic the spiral model: the central nucleus is modified by generating dentritic striae and evolves to form spicules in an effective and 
anarchic way to collect a quantity maximum blood supply from the capillaries of neo-angiogenesis, necessary for growth. We have no definite answer, but it is possible to cite studies of some Scientists.

In 1875 a botanist mathematician by the name of Julius von Wiesner [16] provided a mathematical proof that the helical arrangement of the leaves of a branch in Fibonacci proportions was an effective way to collect a maximum amount of sunlight with a few leaves, he claimed that it was the better way.

After Wiesner's pioneering work, in 1907 a famous dutch botanist Gerrit van Iterson [17], tried to mathematically model the phyllotaxis with the so-called van Iterson model.

We describe our hypothetical model following the van Iterson scheme, simple reticulum: there are infinite propellers that connect successive points in serial order, the shorter of these is called the generating helix highlighted in part (a) (Fig. 4), where the flat reticulum is shown on the universal coating obtained by "unrolling the cylinder", while in part (b) the reticulum is generated from three distinct helices.

This helix is characterized by two parameters, the angle $\mathrm{x}$ called by botanists "the angle of divergence" and their vertical distance y, called "internodal distance".

The tract of generating helix, will wrap around the cylinder a number of times, with the so-called (encyclic number) $\Delta \mathrm{m}(\mathrm{x})$, thus established by van Iterson, defining the secondary divergence: $\delta \mathrm{m}(\mathrm{x})$.

As observed by van Iterson's analysis, this model can provide valuable insights on modeling on biological organisms and in this case on the hypothesis of development of breast cancer.

Recently, 1988 a botanist from Cornell University, Karl Joseph Niklas [18] decided to try this hypothesis in his laboratory.

He discovered that almost every reasonable arrangement of the leaves has the same radiation capacity as the sun.

The crux of the spiraling breast cancer growth model is based on extensive investigations into the influence of gravity on organisms, in particular to the knowledge of the relations between gravity and directional relationships of plants.

In fact Julius von Wiesner published in 1902 an important research, Studien über den Einfluss der Schwerkraft auf die Richtung der Pflanzenorgane [19], a study on the influence of gravity on the direction of plant organs. We have deepened our research and therefore shaped a connection with this important study.

In fact we think that the force of gravity with its action can have an important role on the growth movement that produces the neoplastic spiral, through positive gravitropism and negative gravitropism.

Positive gravitropism allows the neoplasm to develop a complex network of blood vessels consisting of very permeable arteries, veins and capillaries, developing the "vascular system of cancer" forming roots in deep tissues, while negative gravitropism would provide the necessary input to the dynamics of growth.

The negative geotropic curvature favors the growth, which hangs towards the stronger spraying side of the new neoangiogenesis capillaries, activating in sequence many factors that regulate the proliferation and maturation of endothelial cells and vessel wall cells.

Blood vessels are known to change their characteristics in response to the needs of different organs. There is a "dialogue" between the cells of the perfused organ and the vascular cells that determines a high specialization of the latter that adapt to the specific functions of the organ. In cancer things go in a similar way tumors not only induce the formation of new vessels, but according to our studies, they undergo the activity of a very important factor: the action of gravity $(\mathrm{g})$.

The tumor vessels are altered in their structure, potentially frail and very permeable, the action of gravity could modify in an aberrant way the structure and properties, allowing cancer cells to enter the circulation and disseminate [20].

Many studies on plants state that gravity has major effects also on the reticulum of electrical activity of plants, acting on action potentials (AP) and on the plasma membrane (PM) [21]. It has been shown in a major 2006 study by Stankovic [22] that voltage-dependent ion channels and proton pumps (electrogenic $\mathrm{H}+$ - ATPase) are involved in the generation and maintenance of these bioelectric potentials.

Hanke [23] et al. from their study found that plant's AP are sensitive to gravity in the same way they were reported for animal's AP.

We would be propensity to somehow assume this dynamic state that the gravity acts as mechanical stress on the bioelectrical and genetic processes of the cells that together with neo-angiogenesis they propel this structural imbalance tuwards tumor growth.

In fact, every mathematical-physical model needs some assumptions about the phenomenon to be modeled.

A mathematical model is never neutral, objective: there is always a subjective component that reflects the opinions of those who put it into being. If then the predicted consequences of the mathematical-physical-botanical model agree with those that actually manifest themselves in nature, that is, if the model has a good predictive and / or descriptive capacity, somehow they confirm the hypotheses made at the outset.

Mammary gland could represent an interesting model of human fractal geometry [24] where the phyllotactic rules can be reasonably applied and where deviation from normality might give rise to dysfunctions.

We believe, according to theory of spiral cancer, that the neoplastic breast lesion could follow the same model of the Phyllotaxis, Golden Ratio and the Fibonacci sequence, drawing growth and expansion energy from the blood capillaries and by the imbalance of the bioelectric activity of the cells with influenced by the gravity (g) (Fig. 5).

Using golden ratio and Fibonacci sequence, applied to ultrasonography and mammography, we have experimented a diagnostic map with characteristics of high probability of identify suspicious malignancy C4-C5 lesions an early stage.

We detected a different number if lesions with PhiФBreast Imaging and were classified according to BI-RADS descriptors for US imaging, including morphologic features that had a high predictive value for the malignancy $(\mathrm{p}<0.001)$. 
The discriminating capacity of PhiФBreast was found significantly better than normal ultrasonography $(\mathrm{P}<0,05)$.

Furthermore with a predictive algorithm associated with malignant cytology after FNAC, we have classified different types of tumor potentially life threatening for patients.

\section{Discussion}

The history of this important research called PhiФBreast was born in 2016.

Fascinated by botanical and mathematical laws of the phyllotaxis, golden ratio and Fibonacci sequence, we have devised a new diagnostic imaging model to early detection nodular lesions with echostructural features of malignancy.

We have devise this diagnostic mapping model called Phi $\Phi$ Breast applied to ultrasonography and mammography, to identify with high predictive value (PPV) lesions of category C4-C5 of the breast and also gave rise to the elaboration of a modern predictive algorithm and to the model spiral growth of cancer.

A Toshiba Aplio 500 ultrasound system with high frequency probes was used, $10-15 \mathrm{MHz}$ for the study of echostructural changes in the breast.

After considerable study on 987 patients, 55 nodular lesions were identified using the PhiФBreast ultrasonography method for topographic mapping and taking into consideration the following ultrasound features: shape, echostructure, level of echogenicity, margins, size and topographical area.

Lesions detected showed two forms with an markedly hypoechoic echostructure: round shape with irregular and infiltrative margins and cuneiform shape with blurred margins. All features with high predictive value for malignancy $(p<0.001)$.

This diagnostic technique PhiФBreast in ultrasonography and mammography in respecting to the criteria with the American College of Radiology (ACR) [25] \& Breast Imaging Report Data System (BI-RADS) [26] proved to be reliable, validating the early identification of malignant lesions with a high positive predictive value (PPV) with a sensitivity [95\%] specificity [97\%] value positive predictive [97\%] negative predictive value [96\%] comparing our research with important magnetic resonance imaging (MRI) studies performed by various researchers $[34,35,36,37,38]$.

Of the patients selected 55 were subjected to a diagnostic deepening with mammography exam using as a reading interpretation to mammogram the PhiФBreast imaging method and subsequent FNAC [27] under ultrasound guidance using a 19 gauge needle by execution of three passages throught the nodular lesion.

Suspicious lesions detected in to topographic mapping were all category C4 - C5.

The surgical procedure of choice was chosen in the preoperative phase based on the result of the BI-RDAS cytological classification and the study of the udders and lymph node stations of the axillary cables with an MRI examination using contrast medium. Patients were treated with different surgical techniques.
The Veronesi [28] quadrantectomy represents the milestone in the treatment of breast cancer, and currently, it is the first conservative protocol scientifically validated.

The Nipple Sparing Mastectomy (NSM) [29], the Skin Sparing Mastectomy that includes the Nipple - Areola Complex (SSM + NAC) [30] with lymphadenectomy and Intraoperative Radiation Teraphy (IORT) [31].

Post-operative histological findings were all classified as carcinomas.

Particular attention was paid to the ability of PhiФBreast to offer an innovative topographic diagnostic imaging of suspicious lesions and using a predictive algorithm.

Different types of carcinomas have been discovered in the areas of the Golden Spiral, using the golden numbers in the composition of the classification and identified a percentage of probability, according to the relationship between the numbers and consecutive terms of the Fibonacci sequence.

We have utilizing this new nomenclature called "Trapanese's section".

The percentage of tumors detected in the section was as follows: TS1-TS2 [10\%] of ductal carcinomas in situ; TS3TS4 [21\%] of invasive ductal carcinomas; TS5 [14\%] invasive lobular tumors and TS6 [55\%] invasive carcinomas not otherwise specified.

These identified lesions had a high predictive value for malignancy $(\mathrm{p}<0.001)$.

We hypothesized in the analysis to the model spiral breast cancer, the possibility of the existence of different growth sequences, although with the same recursive structure, but due to changes in the conditions of genetic, environmental factors, to alterations of the potentials electrical cell membrane and the action of the gravity, it is possible the hypothesis of development "abnormal morphologies" form of dentritic filaments that allow the expansion of the tumor by recruiting nourishment and energy from the blood capillaries of neoangiogenesis.

Purnell et al. in a recent study revealed the presence of $\Phi$ in human erythrocyte, cell shape, growth and arrangements [32]. Some researchers they showed that in phatological conditions these ratios depart from $\Phi[33]$ and that this angle increases in $138^{\circ}$ (similar to the Golden Angle, 137, $5^{\circ}$ ) generating abnormal growth.

A meticolous study was also performed on the echostructural characteristics of the lesions identified by this mapping, validating that the irregular and spiculated margins are associated with greater probability of malignancy, as also described by other authors.

Liberman [34] et al. described in a study that a spicular margin was the most suspicious characteristic identified with a high PPV.

Wedegärtner [35] et al. reported an irregular margin of the lesion to be the most reliable morphological feature to indicate malignancy.

Schnall [36] and collegues identified spiculated margins to be a highly predictive feature of the cancer image and Gutierrez [37] et al. found irregular or spiculated margins conferring the highest probability of malignancy by BI-RADS classification. 
In a retrospective study, Tozaki [38] and collaborators found irregular shape (97\%) and spiculated margins (100\%) among the features with higher predictive value for carcinoma.

In relation to these important results, we believe that the nodular lesions identified at an early stage using this innovative Phi $\Phi$ Breast imaging method were found to be strongly associated with malignancy $(\mathrm{p}<0.001)$ and with high PPV: $97 \%$.

Wiesner's Law and Da Vinci Divine Proportion have given life to this amazing research.

The scientist does not study nature because it is useful to do it, he studies it because he derives pleasure from it; and get pleasure from it because it is beautiful. If nature were not beautiful, it would not be worth knowing and life would not be worth living as observed by the great scientist Jules Henry Poincare in the passage of Science et méthode 1908 considered the last Universal Genius [39].

\section{Conclusion}

Availing Golden Ratio (Ф), Fibonacci sequence and Predictive Algorithm applied to ultrasonography and mammography, we have given rise to a new diagnostic imaging model called PhiФBreast for the identification of category C4-C5 lesions with high PPV in respecting the criteria of the American College of Radiology (ACR) and Breast Imaging Reporting Data System (BI-RADS).

This original scientific paper could brought progress in science, an important advancement and discovery which will save more life from despair and certain death.

Early diagnosis is essential because it allows the removal of small tumors and therefore able to produce metastases in a more limited number compared to the potential of larger tumors. The amazing theory of spiral cancer, obviously with further insights of hers mechanisms, could be a exceptional method to increase efficacy and precision of future breast cancer diagnostic applications, lead the way toward new therapies such as the use of microgravity that has shown to impose various effects on breast cancer cells.

Microgravity $(\mu \mathrm{g})$ could be utilize as a new anti-tumor technology and therapeutic method in the near future.

PhiФBreast might be the cornerstone of important diagnostic imaging applications as a new strategic weapon against breast cancer.

\section{Acknowledgments}

The authors thank for their support: Foundation T. \& L. de Beaumont Bonelli for Cancer Research - Naples - Italy. CMM Diagnostic Center - Italy.

\section{Author's contributions}

This work was carried out in collaboration among the authors. Author ET prepared and wrote the manuscript, conceived imaging, he developed concept and ideas by the Theory of Spiral Cancer and PhiФBreast Model. Author GT contributed the supervised the manuscript.

\section{Funding}

No funding was received.

\section{Availability of data and materials}

The datasets used during the present study are available from the corresponding author upon reasonable request.

\section{Declarations}

\section{Ethics approval and consent to partecipate}

Not applicable.

\section{Consent for publication}

Not applicable.

\section{Competing interests}

The authors declare that they have no competing interests.

\section{References \\ 1.Siegel RL, Miller KD, Jemal A. Cancer statistics 2015. CA Cancer J Clin. 2015;65:5-29.}

2.Statistical Research and Applications Branch, National Cancer Institute. DevCan: Probability of Developing or Dying of Cancer. DevCan software,version 6.7.3.(2015). Statistical Research and Applications Branch, National Cancer Institute.

3.Tarro GF, Tarro G. Cancer should be only a zodiac sign. Naples, March 2015.

4.Narod SA, Salmena L. BRCA1 and BRCA2 mutations and breast cancer. Discov Med 2011;12:445-53.

5.Ariffin $\mathrm{H}$ et al. Whole-genome sequencing analysis of phenotypic heterogeneity and anticipation in Li-Fraumeni cancer predisposition syndrome Proc. Natl. Acad. Sci.2014. USA, 111,15497 - 15501

6.Leroy B et al. The TP53 website:an integrative resource centre for the TP53 mutation database and TP53 mutant analysis .Nucleic Acids Res. 2013. 41, D962 - D969.

7.Barrow E, Hill J, Evans DG. Cancer risk in Lynch Syndrome. Fam. Cancer 2013;12:229 - 240.

8.Chlebowski RT, Chen Z, Anderson GL, et al. Ethnicity and breast cancer: factors influencing differences in incidence and outcome. J Natl Cancer Inst 2005;97:439-448.

9.Fletcher AS, Erbas B, Kavanagh AM, Hart S, Rodger A, Gertig DM. Use of hormone replacement therapy (HRT) and survival following breast cancer diagnosis. Breast 2005;14:192-200.

10.Tamargo R. J, Pindrik J. A. Mammalian Skull Dimensions and Golden Ratio. J Craniofac Surg 2019;30: 1750-1755.

11.Yetkin G, Sivri N, Yalta K, et al. Golden Ratio is beating in our heart. Int J Cardiol 2013; 168:4926-4927. 
12. Livio M. The Golden Ratio: The Story of Phi, the World's Most Astonishing Number. New York, NY: Broadway Books, Random House Inc; 2002.

13. Vajda S. Fibonacci and Lucas numbers, and the Golden section: theory and applications. Dover Publication 2008; New York, NY.

14.Adler I., Barabé D., Jean R.V., .A History of the Study of Phyllotaxis., Annals of Botany 80,1997.

15.Barlow, P. W. Gravity perception in plants: a multiplicity of systems derived by evolution? Plant Cell Environ 18, 951962 (1995).

16.Wiesner J. Der Lichtgenuss der Pflanzen. Photometrische und physiologische Untersuchungen mit besonderer Rücksichtnahme auf Lebensweise, geographische Verbreitung und Kultur der Pflanzen, Leipzig 1907. Digitalisiert, Aufruf 19.1.2014.

17.Iterson G van. 1907. Mathematische und MikroskopischAnatomische Studien $\mathrm{uX}$ ber Blattstellungen nebst Betraschtungen $\mathrm{uX}$ ber den Schalenbau der Miliolinen. Jena: GustavFischer.

18. Niklas KJ. 1988. The role of phyllotactic pattern as a 'Developmental Constraint' on the interception of light by leaf surfaces. Evolution 42: $1 \pm 16$.

19.Julius von Wiesner. 1902. Studien über den Einfluss der Schwerkraft auf die Richtung der Pflanzenorgane Sitzungsberichte der Akademie der Wissenschaften mathematisch-naturwissenschaftliche Klasse. 111: 733-802.

20.Bijeljic B., Markicevic B., Navaz HK: Capillary climb dynamics in the limits of prevailing capillary and gravity force. Phys Rev E Stat Nonlin Soft Matter Phys 2011;83(5 $\mathrm{Pt}): 056310$

21.Meissner K., Hanke W. Action potential properties are gravity dependent. 2005. Microgravity Sci Technol. $17(2): 38-43$.

22.Stankovic' B. Plant electrophysiology.2006. Springer verlag. Berlin Heidelberg.

23.Hanke W, Fernades de Lima VM, Wiedemann M, Meissner K. Microgravity dependence of excitable biological and physicochemical media. Protoplasma 2006; December; 229 (2-4): 235-42.

24.Moscarelli M, De Paulis R. The Phyllotaxis of the aortica valve. Monaldi Archives for Chest Disease 2019; 89:1139.

25.Mainiero MB, Moy L, Baron P et al. ACR Appropiateness Criteria Breast Cancer Screening. J Am Coll Radiol. 2017;14(11S):S383-S390.
26.Berg WA, Campassi C, Langenberg P et al. Breast Imaging Reporting and Data System Inter-and Intraobserver Variability in Feature Analysis and Final Assessment. AJR Am J Roentgenol.2000;174(6):1769-77.

27.Yu YH, Wei W, Liu JL. Diagnostic value of fine-needle aspiration biopsy for breast mass: A systematic review and meta-analysis. BMC Cancer 2012;12:41.

28.Zurrida S, et al. The Veronesi quadrantectomy: an established procedure for the conservative treatment of early breast cancer. Int J Surg Investig 2001;2(6):423-31.

29.Crowe, J.P. Jr., Kim, J.A., Yetman, R., Banbury, J., Patrick, R.J., Baynes, D. Nipple-sparing mastectomy: technique and results of 54 procedures. Arch Surg 2004;139:148-150.

30.Gerber, B., Krause, A., Reimer, T. et al, Skin-sparing mastectomy with conservation of the nipple-areola complex and autologous reconstruction is an oncologically safe procedure. Ann Surg 2003;238:120-127.

31.Sedlmayer, F., Reitsamer, R., Wenz, F. et al, Intraoperative radiotherapy (IORT) as boost in breast cancer. Radiat Oncol 2017;12:23.

32.Purnell MC, Butawan MBA, Ramsey RD. Bio-field array: a dielectrophoretic electromagnetic toroidal excitation to restore and maintain the golden ratio in human erythrocytes. Physiol Rep 2018;6:e13722.

33.Henein MY, Zhao Y, Nicoll R, et al. The human heart: application of the golden ratio and angle. Int J Cardiol 2011; 150:239-242

34.Liberman L, Morris EA, Lee MJ-Y, et al. Breast lesions detected on MR imaging: features and positive predictive value. AJR Am J Roentgenol 2002;179(1):171-178.

35.Wedegärtner U, Bick U, Wörtler K, Rummeny E, Bongartz G. Differentiation between benign and malignant findings on MR-mammography: usefulness of morphological criteria. Eur Radiol 2001;11(9):1645-1650.

36.Schnall MD, Blume J, Bluemke DA, et al. Diagnostic architectural and dynamic features at breast MR imaging: multicenter study. Radiology 2006;238(1):42-53.

37.Gutierrez RL, DeMartini WB, Eby PR, Kurland BF, Peacock S, Lehman CD. BI-RADS lesion characteristics predict likelihood of malignancy in breast MRI for masses but not for nonmasslike enhancement. AJR Am J Roentgenol 2009;193(4):994-1000.

38.Tozaki M, Igarashi T, Fukuda K. Positive and negative predictive values of BI-RADS-MRI descriptors for focal breast masses. Magn Reson Med Sci 2006;5(1):7-15.

39.Henri Poincaré. Science et méthode (1908), Flammarion, Paris, 1920. 

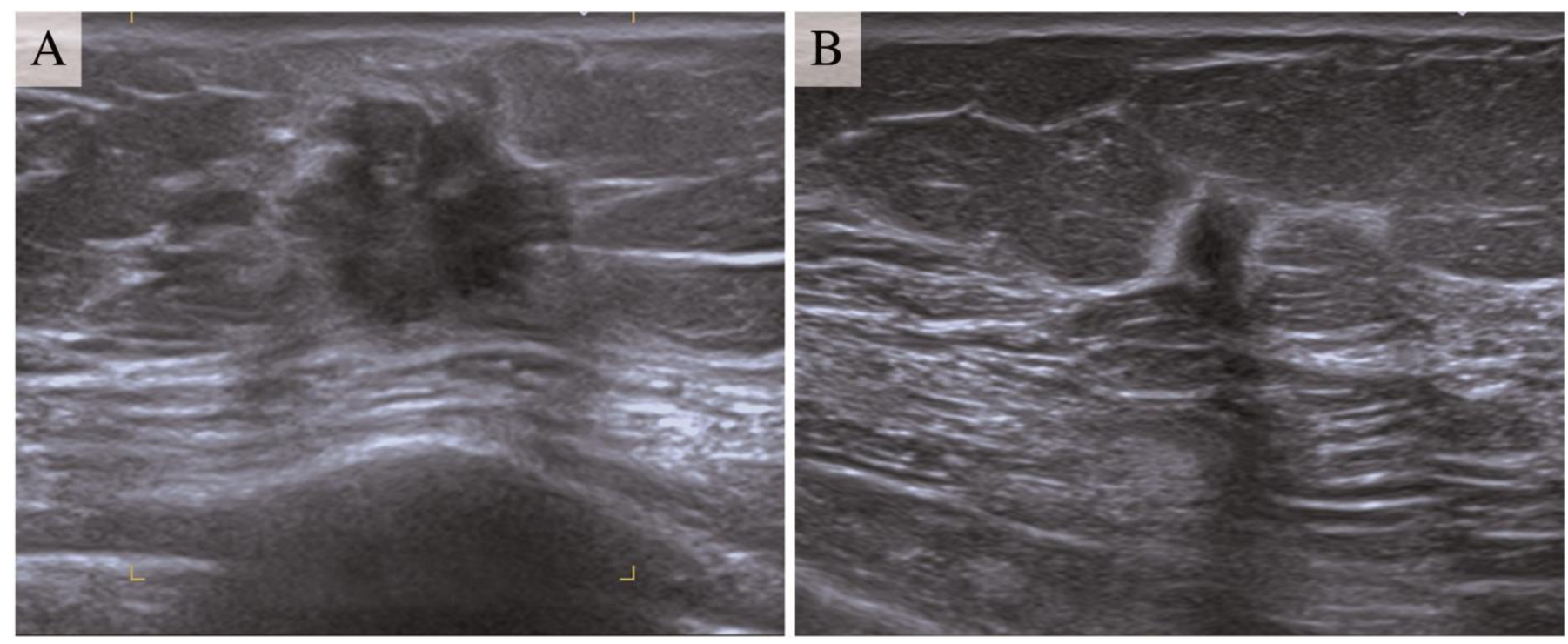

Fig. 1 Ultrasonography Image Of Suspicious Nodular Lesions Identified With PhiФBreast Model

Solid lesion, round shape, markedly hypoechoic echostructure with irregular and infiltrative margins (Panel A). Solid lesion, cuneiform shape, markedly hypoechoic echostructure with blurred margins (Panel B). 

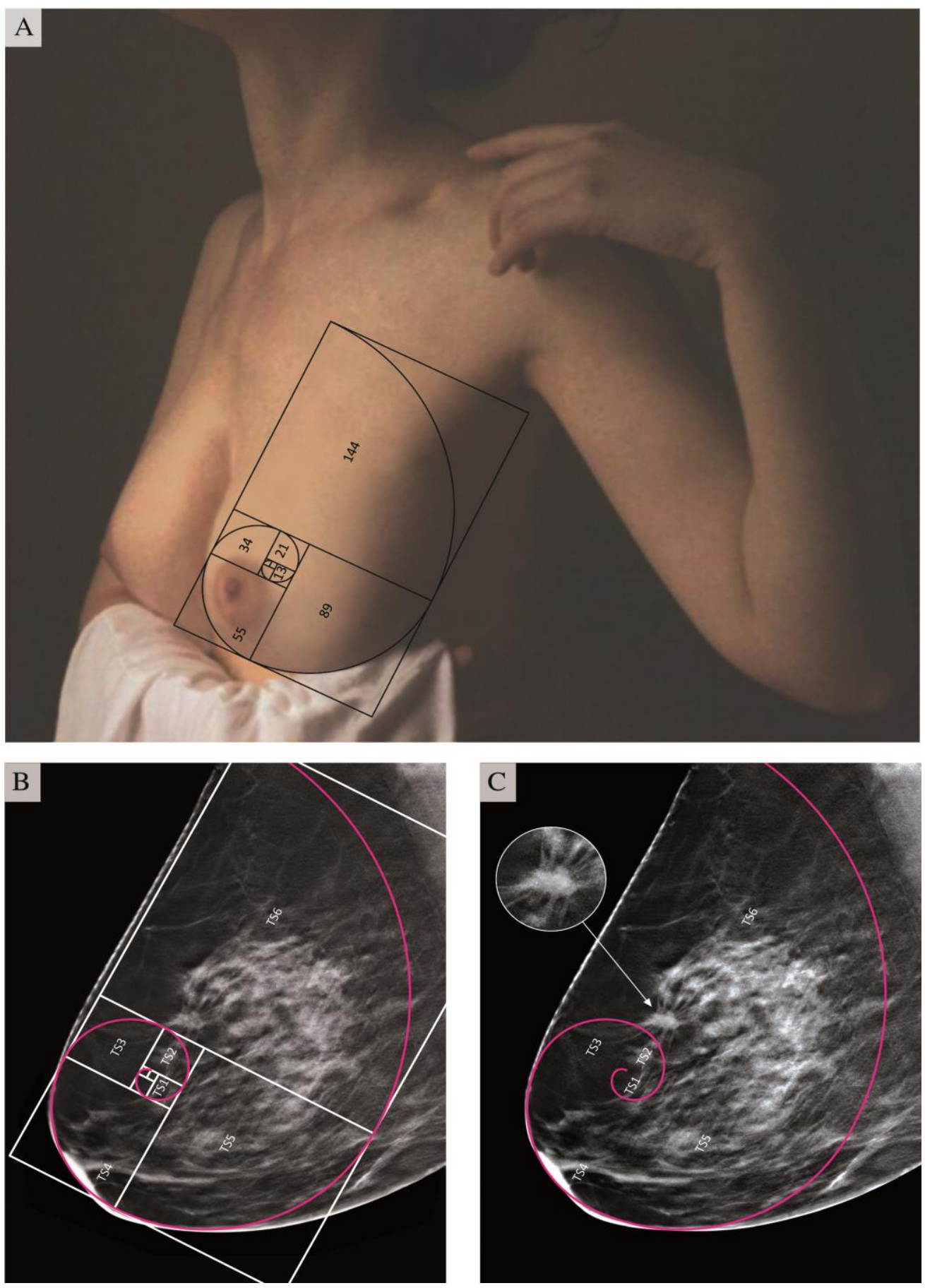

Fig. 2 PhiфBreast A New Diagnostic Technique For Breast Cancer Detection

Panel A show the fascinating symmetry of the breast that ultimately can be rapresented by Golden Spiral inscribed in the regular breast anatomy. Panel B show mediolateral oblique view (MLO) of the breast at mammographic x-ray with applied Golden Ratio and Fibonacci sequence. Panel C Phi $\Phi$ Breast imaging applied to the mammogram has provided a mapping of neoplastic lesion. White arrow highlights cancerous tumor detected in TS6 section of the Golden Spiral. Cancerous mass appear as a bright and irregular image with spiky edges. 


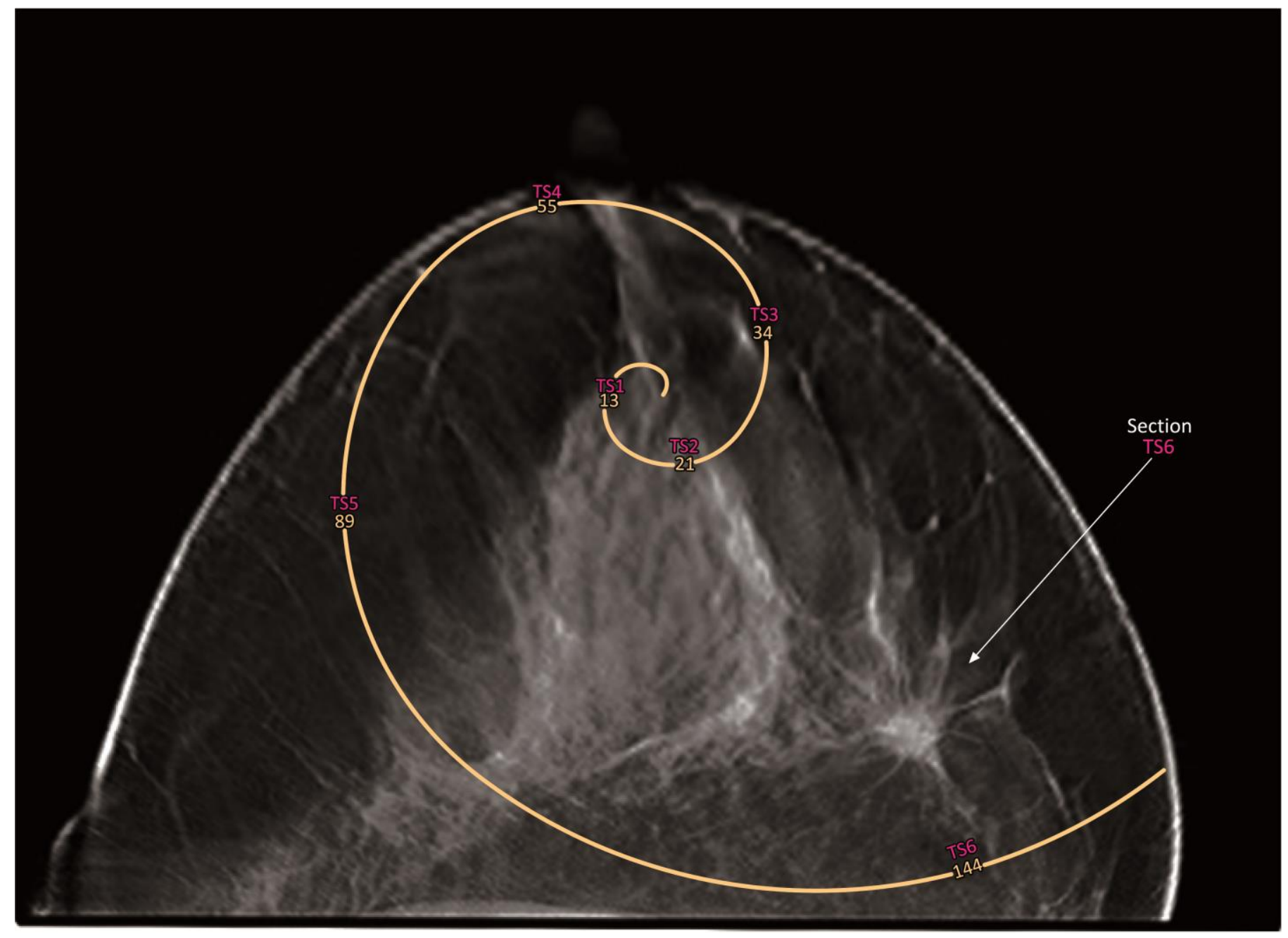

Fig. 3 Golden Ratio $(\Phi)$ Breast Cancer Detection And Fibonacci Sequence

Cranial-caudal view (CC) show Fibonacci Spiral approximates the Golden Ratio $(\Phi)$ using mammogram inscribed in squares of integer Fibonacci-number side, shown for square size $(13,21,34,55,89,144)$ making use of a new nomenclature called “Trapanese's Section TS1,TS2,TS3,TS4,TS5,TS6”. The arrow show extraordinary x-ray vision of the cancer detected in TS6 section. 


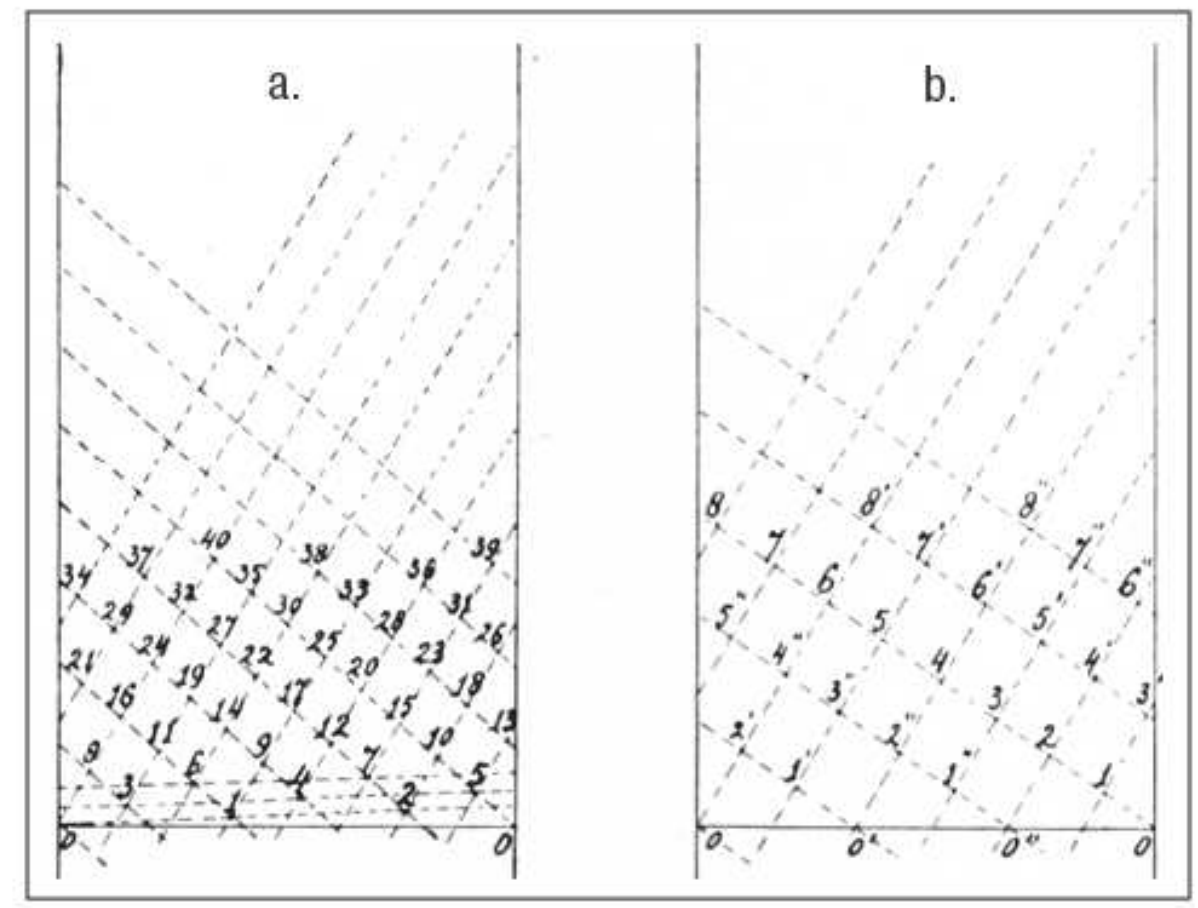

Fig. 4 The Mechanical Model Of Van Iterson

\section{Table 1. Predictive Algorithm Breast Cancer}

Histological types and percentage tumors detected in the Golden Ratio (Ф)

\begin{tabular}{|lll|}
\hline${ }^{\Phi}$ Section Area 13 & *TS1 Ductal Carcinomas in Situ & $3 \%$ \\
\hline${ }^{\Phi}$ Section Area 21 & *TS2 Ductal Carcinomas in Situ & $7 \%$ \\
\hline${ }^{\Phi}$ Section Area 34 & *TS3 Invasive Ductal Carcinomas & $13 \%$ \\
\hline${ }^{\Phi}$ Section Area 55 & *TS4 Invasive Ductal Carcinomas & $8 \%$ \\
\hline${ }^{\Phi}$ Section Area 89 & *TS5 Invasive Lobular Tumors & $14 \%$ \\
\hline${ }^{\Phi}$ Section Area 144 & *TS6 Invasive Carcinomas not Otherwise Specified & $55 \%$ \\
\hline
\end{tabular}

${ }^{\oplus}$ Golden Ratio and Fibonacci sequence *New Nomenclature Trapanese's Section

${ }^{\Phi}$ Golden Ratio And Fibonacci Sequence With The New Nomenclature *Trapanese's Section

Percentage of tumors detected in the sections: TS1-TS2 $10 \%$ of ductal carcinomas in situ; TS3-TS4 $21 \%$ of invasive ductal carcinomas; TS5 14\% invasive lobular tumors and TS6 55\% invasive carcinomas not otherwise specified. 

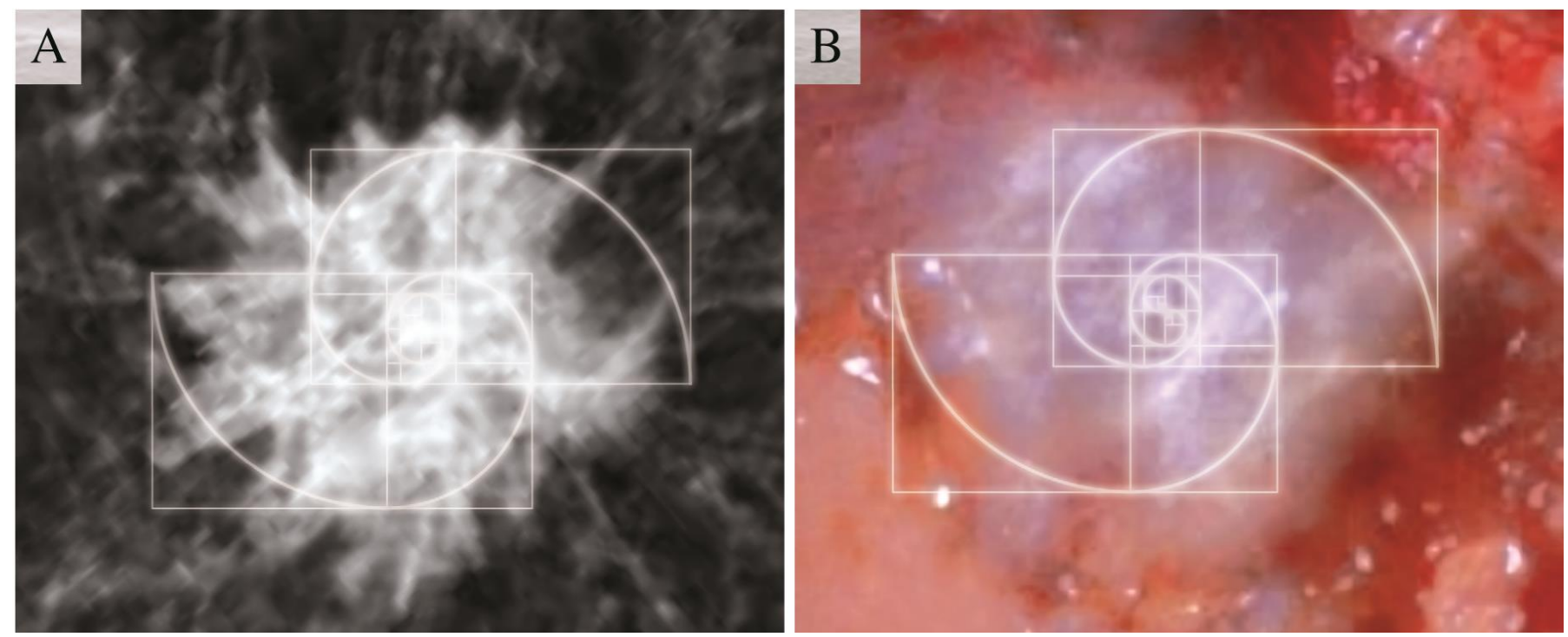

Fig. 5 Mammography Breast Cancer By PhiФBreast Pattern \& Spiral Tumor Growth Imitating The Golden Ratio Model (Ф) Mammographic X-ray seen on a closeup view large spiculated carcinoma projected over a background primarily of soft tissue parenchyma as spiral growth pattern of breast cancer in perfect configuration with the Golden Ratio $(\Phi)$ and Fibonacci Spiral (Panel A).

Fresh tissue specimen of the resected breast area and the tumor within. Extraordinary image showing the tumor growth imitating the Golden Ratio model $(\Phi)$ with a different fractal geometry, generating an abnormal spiral infiltrating healthy breast tissue due to the neoangiogenesis, imbalance of the bioeletric activity of the cells and the action of gravity (g) (Panel B). 